\title{
Combined Nearest Greedy Algorithm With Randomized Iterated Greedy Algorithm to Solve Waste Collection Problem
}

\author{
Abdulwahab Almutairi \\ Correspondence: Abdulwahab Almutairi, School of Mathematics, Unaizah college of Sciences and Arts, Qassim \\ University, Qassim, Saudi Arabia. E-mail: a.abdulwahab.a@gmail.com
}

Received: March 8, 2020 Accepted: April 15, 2020 Online Published: April 25, 2020

doi:10.5539/ijsp.v9n3p66 URL: https://doi.org/10.5539/ijsp.v9n3p66

\begin{abstract}
The waste collection considers as one of the common transportation problems in the operation research and management area and also it is a significant activity in each city. In this kind of the paper, we study a version of a real-life waste collection and try to find an efficient way to reduce the costs such as the cost of the operation such as fuel and maintenances, the cost of the environment such as noise and traffic congestions, the cost of the investment such as vehicles fleet. The waste collection problem can be formulated as a well-known Vehicle Routing Problem (VRP). The basic idea is to attempt to develop a daily truck routing which will improve the efficiency of the vehicle distribution in Riyadh. The solution will be done in a good way that it can serve all the customers, while in the meantime, it will attempt to improve the total cost. The main contribution of this paper is to improve the solution of total costs for the waste collection while using the existing resources through the combination of the Nearest Greedy algorithm with both Iterated Greedy (IG) and Randomized Iterated Greedy (RIG). We execute our proposed method with real data that collect waste from more than 100 customers in Riyadh city. In terms of the experiments, the results received by those methods are successfully implemented and improved the overall waste collection in Riyadh. In conclusion, these algorithms able to reduce the total costs to this kind of case study with the same number of vehicles.
\end{abstract}

Keywords: waste collection, local search, iterated greedy, randomization

\section{Introduction}

The vehicle routing problem considered one of the most important problems in the class of NP-hard problems. Nowadays, the research societies have been proposed many of the heuristics techniques in order to solve the rising issue which is the waste collection as well as make better use of their resources. Cities are faced with several problems such as a high volume of waste, the costs, and the impact of the waste on both global and local environments. Many cities across the world have proposed and implemented optimization techniques for their waste collection scenarios, which are usually very different from each other. The waste collection problem can be described as routing vehicles in order to collect many customers' waste and return to the central depot with minimizing travel total cost. This kind of problem is known in many papers as the Waste Collection Vehicle Routing Problem. The fundamental aim of waste management is to reduce and eliminate the adverse impacts of waste materials on human health and the environment in order to support economic development and a better quality of life. The paper is organized as follows: Section 2 describes the logistic systems in waste collection: Riyadh case description. In Section 3 we present an overview of a literature review. Section 4 presents an overview of the proposed algorithm. Section 5 shows the results of applying the proposed methodology to a real context case in Riyadh. To conclude, Section 6 summarizes with recommendations.

\section{Logistics Systems in Waste Collection: Riyadh Case Description}

The data of the waste collection is confined to the capital of Saudi Arabia (Riyadh) with an area of 380,000 square Kilometers. Consequently, Riyadh occupies $19.5 \%$ of the total area of the Kingdom of Saudi Arabia and it is the second-largest city in Saudi Arabia in terms of area after the Eastern Province. Riyadh is divided into a number of governorates, which are divided into categories (A) and (B), and the number of 12 provinces of category A and eight provinces of category B. Riyadh includes a number of centers that are administratively linked to it and there are 172 centers of category A and 282 centers of category B, which is the largest areas of Riyadh.

It is known that the quantities of waste generated and the required cleaning works are directly proportional to what happens in any city in terms of population density and urbanization. Naturally, the city of Riyadh has a rapid population growth and heavy urbanization in all directions, it is normal and expected to increase annually the rate of waste output in the city and accordingly cleaning efforts required to be increased. The amount of waste will stabilize only with the 
growth of the city population and urbanism.

The municipal solid waste landfill is considered an essential means for the disposal of waste and also is of great importance and sensitive at all levels. This holds the concern and its employees responsible for drawing up current and future plans, preparing the necessary studies, and implementing projects to absorb the disposal of the quantities of waste generated in the city in safe ways and a modern sophisticated approach for many decades to come.

The municipal solid waste landfill of Riyadh is located in the south-east of the Al-Sulay area, about $25 \mathrm{~km}$ from the city center. It is intended for the reception of municipal solid waste only, and it is prohibited to receive medical, industrial and hazardous waste of all kinds. The total area of the entire landfill area is (10) million m2. The municipal solid waste landfill has a length of more than $11 \mathrm{~km}$ and is surrounded by a protective fence. As the only municipal solid waste landfill in Riyadh, it receives about 2,000 vehicles per day, carrying more than 16,000 tons of various types of municipal solid waste, construction waste per day. Note that there are (4) surface scales, each of which has a capacity of up to (100) tons and always one of them alternately in reserve mode.

\subsection{Waste Disposal}

In a municipal landfill, waste is disposed of by dumping it in a fixed length and height space called a cell. These cells are separated from each other by a thin layer of soil called a cell cover (coverage) with a thickness of about $10 \%$ of the cell height. In the municipal landfill in Riyadh, the length of each cell is $170 \mathrm{~m}$, width is $100 \mathrm{~m}$, height is $3 \mathrm{~m}$, and the cell cover is $30 \mathrm{~cm}$. The landfill consists of vertical layers and each layer consists of a number of horizontal cells. There is a large number of cell forms, and the cell model used in any layer of a municipal landfill and its arrangement during implementation depends on the appropriate operating plans for the landfill. The most common cell models are the following:

- Parallel Form: Conducts adjacent and parallel cells, coverage tracking (inflicting) the burial process at half the length of the cell under operation

- Reciprocal model: Operation depends on the execution of non-adjacent cells interchangeably, and the sequential coverage in the previous executing cell.

- The spiral model: the cells are executed during operation in a continuous ascending circular, and the coverage follows (inflicts) the burial process at a distance within the length of the hemisphere.

- Single-cell model: use the entire burial area as a single operating cell, coverage tracking (inflicted) the burial process is half the distance from an operation.

\subsection{Municipal Landfill Operation Plan}

The operating plan and methods of implementation in the municipal landfill are governed by a number of factors, the most important of which are:

- The total area of the municipal landfill and the topography of the general site (absorption capacity).

- A number of vehicles that come into the cell per day (in motion).

- The amount of waste received into the cell per day (volume and growth).

- Speed of coverage and compaction ratio (quality of implementation).

- A number of layers of municipal landfill expected by design (shelf life).

The first layer of the existing municipal landfill consisted of 28 cells, and there are signs and flags installed in specific locations, help workers to know the limits of each cell and its height during work. Due to the large quantities of waste reaching the municipal landfill daily, the Riyadh municipal landfill followed the strategy of dividing one cell into a number of segments. Consequently, the cell can accommodate the entire amount of waste (household and commercial) received within a day 24 hours (within eight thousand tons). The cell is covered with soil within a period of not more than 20 hours. This is a standard time to cover the open cell so that it does not take sufficient time for aerobic decomposition and cause waste rot. Thus, landfill cells are composed of cells and layers.

\section{Literture Review}

In this section, we briefly review the literature on VRP with waste collection, especially with a real case study. Nowadays, the collection of waste Vehicle Routing Problem (VRP) has received more attention. As a result, numbers of problems and a wide variety of exact and approximate algorithms have been applied in order to find the optimal solution.

(Gruler, et al., 2015) applied a simheuristic for a real-life waste collection with stochastic demands in smart cities. The aim is to develop and describe the method in order to improve waste collection processes. The methodology allows the 
consideration of stochastic waste levels in garbage containers, leading to more realistic and reliable routing solutions in waste management. (Ismail \& Irhamah, 2008) present a real case study of the waste collection with stochastic demands. Case study (Ismail \& Irhamah, 2008) improves the results when minimizing the distance of travel using a hybrid genetic algorithm-tabu search compared with each genetic algorithm and tabu search alone. The data of this problem are inspired by the real case study of the waste collection problem. The experiment results illustrate that the HGATS is good for solving large problem size and it's more robust than GA although the performance of HGATS and TS are similar.

(Faccio, Persona, Zanin 2011) addressed the real case study that knowing the real-time of each vehicle and the real-time waste level of each bin. They present a new multi-objective routing model with real-time in order to optimize the route plan and environment impact and to minimize the number of vehicles and distances travel. The model and simulation have been tested on a set of benchmark data from an Italian city of about 100,000 inhabitants. One of the main results that have been achieved is to allow producing real-time input which is important in the proposed routing model.

(Buhrkal, Larsen, Ropke, 2012) described an adaptive large neighborhood search algorithm for solving the Waste Collection Vehicle Routing Problem with Time Window. Two different real case studies with different requirements have been considered including the lunch and rest break. The meta-heuristic Adaptive Large Neighbourhood Search for solving this kind of problem can illustrate the main advantage which is the usefulness of the algorithm by showing that the algorithm shows the improvement of the objective for benchmark instances from the literature also for instances provided by a Danish garbage collection company were achieved. (Kim, Kim, Sahoo, 2006) attempt to solve the real-life waste collection problems at Waste Management with time windows (VRPTW) and they used the insertion algorithm. The kind of problem can be characterized as a variant of VRPTW and the main variation is due to disposal operations and driver's lunch break. As a consequence, the presented algorithms have been successfully applied and deployed for the real-life waste collection problems of Waste Management. (Mat, Benjamin, Abdul-Rahman, Wibowo, 2017) propose the nearest greedy (NG) technique in order to solve a real-life case study related to the collection of the waste in the northern part of Malaysia. The technique was applied to create an initial route for each area. This kind of real case study consisted of six areas where each involved up to 103 customers. They have compared their results with the waste collection company results within the city. (Mat, Benjamin, Abdul-Rahman, Wibowo, 2017) has been proposed the nearest neighbor algorithm and the greedy algorithm in order to solve the travel salesman problem. In terms of the experiments, the paper has presented different results on known library problems therefore the results achieved by these algorithms are efficient.

\section{Methodology}

This section proposed the combination of two types of algorithms for solving a waste collection problem. The greedy algorithm considers as one of the easy and simplest algorithms to apply in different type of problems because this kind of algorithm can take the nearest/closest/most optimal choice, and repeat this process. The nearest greedy algorithm always chooses a set customers seems to be the best at the moment and then it cannot change the customers at a later point. The greedy algorithm has been proposed in (Benjamin, Beasley, 2011) in order to solve a waste collection vehicle routing problem with time windows. Also, the Iterated greedy algorithm and Randomised Iterated Greedy algorithm have been proposed in (Almutairi, 2018) in order to solve a waste collection in urban area of the capital of Saudi Arabia. We have used the same data that presented in (Almutairi, 2018).

The target of the real case study problem is to minimize the total costs of serving the customers. In this paper, the Nearest Greedy approach had been applied in order to build vehicle routes to solve a real case study of the waste collection problem. Total number of customers for each area can be calculated as follow $\mathrm{n}$ (total customers of the area) + one depot (central depot) + one disposal facility. In fact, the driver started the route from node 0 (central depot) with limited vehicle capacity in order to serve customers. After that, the NG algorithm was employed to determine the next closest customer's location from central depot in order to be served by comparing all distances from central depot to $\mathrm{n}$ customer's location. The next step is that algorithm selected the customer location with the shortest distance and then adding to the present route and updated the remaining number of customers' locations to be served. The capacity of the vehicle has to be check after each customer had been served. If the capacity of the vehicle had been fully loaded, the vehicle would travel to unload the waste at central depot. The process repeated until all customers had been served. Note that the driver has to not serve the same customer twice. Before travel back to central depot, the vehicle would have to travel to disposal facility in order to empty its load. The algorithm calculated the total travel distance by the vehicle and displayed the built vehicle route. Finally, the algorithm was terminated.

In terms of the iterated greedy algorithm, the sequence of solutions is generated by using two phases: destruction and construction while in the randomised iterated greedy algorithm, the process of the iterated greedy algorithm is extended by adding the randomisation in order to improve the initial solution for the first phase of the algorithm. The details of each algorithm are explained by (Almutairi, 2018). 


\section{Results and Discussion}

In the computational results, the main performance measure used is the capacity of the vehicles. Besides this, we reported additional measures during the planning horizon: minimize the number of vehicles and utilization for the loading capacity. Therefore, these measures lead to reduce the total costs of the service during the service. This section shows the heuristics applied to the real case study in the capital of Saudi Arabia and reports the obtained experimental results. Table 1 shows for each contract the number of areas. Table 1 shows the results from the Iterated Greedy algorithm (denoted in table 1 by IG), Randomised Iterated Greedy algorithm (denoted in table 1 by IG) and our method. The algorithm's codes are programmed using the Java application. A Macintosh HD on $2.3 \mathrm{GHz}$ Intel HD Graphics 40001024 MB with Intel Core i7 with 4 GB 1600 MHz DDR3 has been used to perform the computational experiments. Table 1 shows the experiments results achieved for the real case study by various methods using IG, RIG and Nearest Greedy algorithm. The proposed heuristics are examined and verified using the benchmarks problem provided by the company in order to improve the solutions. Table 1 presents the combination of computational results for three heuristic algorithms, which are IG, RIG, and our method. Based on the results shown above, it can be concluded that the computational results from our method achieved the best solution in terms of total costs traveled, in comparison to other heuristic algorithms solutions.

Based on the solutions proposed by IG, RIG and our method, table 1 presents an overall description of all 10 contracts in terms of case study data sets and main results. The project has 10 contracts and each contract covers a specific area and the results can be seen in Table 1. The results for a particular instance are presented in the following columns. In the first column, there are 10 contracts in the case study that has been considered. The second column represents how many areas can each contract cover which is called the supervisory area. The number of routes that are used to solve the problem is shown in the third column. The fourth, fifth and sixth columns represent the total costs that have been achieved by using both methods IG, RIG and our method respectively. Moreover, the percentage deviation formula between the approaches is calculated as follows;

$$
\text { Percentage deviation }=\left(\frac{\text { old solution })-(\text { new solution })}{(\text { new solution })}\right) * 100 .
$$

Table 1 . Summary table of 10 contracts investigated in part Riyadh municipality

\begin{tabular}{|c|c|c|c|c|c|c|c|c|c|}
\hline Problem sets & $\begin{array}{c}\text { Supervisory } \\
\text { area }\end{array}$ & Routes & $\begin{array}{c}\text { Total } \\
\text { costs (1) } \\
\text { (IG) }\end{array}$ & $\begin{array}{c}\text { Total } \\
\text { costs (2) } \\
\text { (RIG) }\end{array}$ & $\begin{array}{c}\text { Our } \\
\text { methods } \\
(3)\end{array}$ & $\begin{array}{c}\text { GAP } \\
(1)-- \\
(2)\end{array}$ & $\begin{array}{c}\text { GAP } \\
(1)-- \\
(3)\end{array}$ & $\begin{array}{c}\text { GAP } \\
(2)--\end{array}$ & $\begin{array}{c}\text { Waste } \\
\text { generated } \\
(\mathrm{kg})\end{array}$ \\
\hline Contract code_1 & 8_areas & 98 & 1583.5 & 1533.5 & $\mathbf{1 5 3 2 . 9}$ & $3.26 \%$ & $3.30 \%$ & $0.04 \%$ & 1637.78 \\
\hline Contract code_2 & 13_areas & 150 & 1742.3 & 1695.3 & $\mathbf{1 6 9 3 . 6}$ & $2.77 \%$ & $2.87 \%$ & $0.10 \%$ & 2596.95 \\
\hline Contract code_3 & 7_areas & 90 & 1454.3 & 1428.3 & $\mathbf{1 4 2 7 . 5}$ & $1.82 \%$ & $1.88 \%$ & $0.06 \%$ & 1509.78 \\
\hline Contract code_4 & 14_areas & 158 & 2013 & 1989 & $\mathbf{1 9 8 7 . 6}$ & $1.21 \%$ & $1.28 \%$ & $0.07 \%$ & 3233.36 \\
\hline Contract code_5 & 17_areas & 164 & 2073.5 & 1999.5 & $\mathbf{1 9 9 6 . 5}$ & $3.70 \%$ & $3.86 \%$ & $0.15 \%$ & 2727.69 \\
\hline Contract code_6 & 6_areas & 66 & 1426.5 & $\mathbf{1 3 7 9 . 5}$ & $\mathbf{1 3 9 7 . 5}$ & $3.41 \%$ & $3.41 \%$ & $0 \%$ & 897.68 \\
\hline Contract code_7 & 9_areas & 94 & 1485.2 & 1437.2 & $\mathbf{1 4 3 6 . 8}$ & $3.34 \%$ & $3.37 \%$ & $0.03 \%$ & 1774.6 \\
\hline Contract code_8 & 10_areas & 88 & 1337.5 & 1325.5 & $\mathbf{1 3 2 4 . 2}$ & $0.91 \%$ & $1.00 \%$ & $0.10 \%$ & 1625.5 \\
\hline Contract code_9 & 9_areas & 142 & 1752.6 & 1715.6 & $\mathbf{1 7 1 2 . 1}$ & $2.16 \%$ & $2.37 \%$ & $0.20 \%$ & 2114.45 \\
\hline $\begin{array}{c}\text { Contract } \\
\text { code_10 }\end{array}$ & 7_areas & 93 & 1501.3 & 1499.3 & $\mathbf{1 4 9 8 . 1}$ & $0.13 \%$ & $0.21 \%$ & $0.08 \%$ & 1496.73 \\
\hline Average & ---------- & ------ & 1636.97 & 1600.27 & $\mathbf{1 5 9 8 . 8 8}$ & $2.27 \%$ & $2.35 \%$ & $0.08 \%$ & 1961.45 \\
\hline
\end{tabular}

Generally, the solutions on the case study problem are improved on most of the contracts which are 9 out of 10 contract codes. The gaps are expressed as a percentage improvement value in the distance when compared to two approaches and the respective gaps between total costs are shown in Table 1 under the (7), (8) and (9) column. The gap is generally quite small and does not reach $1 \%$ when we compared our method with RIG. Therefore, the results clearly showed that using our method in those instances able to provide better solutions in average results than the methods presented earlier. In most cases these gaps are small; sometimes it can be zero such as in contract code_6. The last column showed the waste generated for each contract. In Table 1, we summarise the results obtained by the methodologies where the vehicle capacity is considered during the design stage as $100 \%$. Examining table 1, it is clear that our three methods 
generate routes of similar quality. Also, we would be justified in choosing the method that obtained the lowest total costs for all. From the average presented at the bottom of table 1, it is clear that our method is to be preferred, having lower average total costs than both (IG) and (RIG). Also, the best result for each instance is shown in bold. RIG clearly outperforms the IG whereas our methods outperformed both (RIG) and (IG).

\section{Conclusion}

In this case study, methodologies were applied in order to minimize the total costs of the problem. We considered a VRP that arises when a number of known customers have waste that has to be collected by specific vehicles. Empty vehicles have to leave the central depot and follow the initial routes to collect waste from known customers, emptying themselves at waste disposal facilities when it's necessary. We attempted to solve the problem by creating an initial solution in the beginning and assigning each customer to a specific vehicle using these methodologies. Experiment results were done on the case data in Riyadh and these results showed the improvement of the results between these methodologies. For further research side, the time window either soft or hard can be considered in order to improve the work, reduce the cost and improve the quality of the solutions. To do this research, more efforts are required to gather the data that related to the time window.

\section{Recommeddation}

It is certain that the awareness of the community has an active role in raising the level of cleanliness of the city and reduces the amount of waste generated. Given the social habits of Saudi citizens, awareness-raising factors may be more influential in upgrading than in reducing the daily waste rate.

Companies have to provide containers of different shapes and sizes to suit each sector, sector waste buildings and construction containers are large in size, while medium-sized containers have to provide for hotels, companies, and neighborhoods. The goal for medium-sized is to prevent the gathering of flies and insects around. Companies also have to provide small containers placed inside parks, restaurants and hospitals and the goal is to easily put the waste inside by individuals. We also put containers with waste to be recycled. The goal is to put bottles, plastic, metals, and papers inside them for the purpose of developing a culture of recycling waste in Saudi society.

- The development of waste management and recycling and contribute to the preservation of the environment and improve the urban landscape must achieve several goals:

- Implementation of the overall strategy for the waste management sector

- Promote a culture of screening from the source

- Recycling of annual solid waste production

- Recycling annual production of construction waste and demolition

- Addressing the visual distortion of the spread of containers in neighborhoods and streets

- To create a sustainable and healthy environment.

\section{References}

Almutairi, A. (2018). Using randomization into iterated greedy algorithm in order to solve capacitated vehicle routing problem. Journal of Natural Science and Maths. JNM: 2018 MATH-03.

Almutairi, A. (2018). Solving capacitated vehicle routing problem by iterated greedy algorithm. Journal of Natural Science and mathematics. JNM: 2018 MATH-02.

Benjamin, A. M., \& Beasley, J. E. (2010). Metaheuristics for the waste collection vehicle routing problem with time windows, driver rest period and multiple disposal facilities. Computers \& Operations Research, 37(12), 2270-2280. https://doi.org/10.1016/j.cor.2010.03.019

Buhrkal, K., Larsen, A., \& Ropke, S. (2012). The waste collection vehicle routing problem with time windows in a city logistics context. Procedia-Social and Behavioral Sciences, 39, 241-254. https://doi.org/10.1016/j.sbspro.2012.03.105

Faccio, M., Persona, A., \& Zanin, G. (2011). Waste collection multi objective model with real time traceability data. Waste management, 31(12), 2391-2405. https://doi.org/10.1016/j.wasman.2011.07.005

Golden, B. L., Assad, A. A., \& Wasil, E. A. (2002). Routing vehicles in the real world: applications in the solid waste, beverage, food, dairy, and newspaper industries. In The vehicle routing problem (pp. 245-286). Society for Industrial and Applied Mathematics. https://doi.org/10.1137/1.9780898718515.ch10

Gruler, A., Juan, A. A., Fikar, C., \& Hirsch, P. (2015). A Simheuristic for the Waste Collection Problem with Stochastic Demands in Smart Cities. Simulation in Production and Logistics. Markus Rabe \& Uwe Clausen (eds.) Fraunhofer 
IRB

Verlag,

Stuttgart.

https://www.researchgate.net/publication/282247374_A_Simheuristic_for_the_Waste_Collection_Problem_with_S tochastic_Demands_in_Smart_Cities.

Gupte, S. K., \& Bhatia, R. K. (2017). Routing optimization of municipal solid waste collection in Jabalpur city using ARC GIS. International journal of trend in scientific research and development, $2(1), 457$. https://doi.org/10.31142/ijtsrd7000

Ismail, Z., \& Irhamah, I. (2008). Solving the vehicle routing problem with stochastic demands via hybrid genetic algorithm- tabu search. Journal of mathematics and statistics, 4(3), 161-167. https://doi.org/10.3844/jmssp.2008.161.167

Kim, B. I., Kim, S., \& Sahoo, S. (2006). Waste collection vehicle routing problem with time windows. Computers \& Operations Research, 33(12), 3624-3642. https://doi.org/10.1016/j.cor.2005.02.045

Kim, J. D., Choi, H. S., \& Lee, D. H. (2007, August). Vehicle routing in a refuse collection system: a case study. In 2007 International Conference on Computational Science and its Applications (ICCSA 2007) (pp. 467-473). IEEE. https://doi.org/10.1109/ICCSA.2007.12

Kizilateş, G., \& Nuriyeva, F. (2013). On the nearest neighbor algorithms for the traveling salesman problem. In Advances in Computational Science, Engineering and Information Technology (pp. 111-118). Springer, Heidelberg. https://doi.org/10.1007/978-3-319-00951-3_11

Mat, N. A., Benjamin, A. M., \& Abdul-Rahman, S. (2018). Efficiency of heuristic algorithms in solving waste collection vehicle routing problem: a case study. The Journal of Social Sciences Research, (SPI6), 695-700. https://doi.org/10.32861/jssr.spi6.695.700

Mat, N. A., Benjamin, A. M., Abdul-Rahman, S., \& Wibowo, A. (2017, November). Nearest greedy for solving the waste collection vehicle routing problem: A case study. In AIP Conference Proceedings (Vol. 1905, No. 1, p. 040018). AIP Publishing LLC. https://doi.org/10.1063/1.5012206

Moustafa, A., Abdelhalim, A. A., Eltawil, A. B., \& Fors, N. (2013). Waste collection vehicle routing problem: case study in Alexandria, Egypt. In The $19^{\text {th }}$ International Conference on Industrial Engineering and Engineering Management (pp. 935-944). Springer, Berlin, Heidelberg. https://doi.org/10.1007/978-3-642-37270-4_89

Rajan, L., Saji, K. S., \& Saraswathi, U. (2018). Solid waste management using internet of things and android application. GRD Journal/ Global research and development journal for engineering/ national conference on emerging trends in electrical, electronics and computer engineering (ETEEC-2018). E-ISSN: 2455-5703.

Ruiz, R., \& Stützle, T. (2005). An iterated greedy algorithm for the flowshop problem with sequence dependent setup times. In The 6th Metaheuristics International Conference (pp. 817-826).

Ruiz, R., \& Stutzle, T. (2007). A simple and effective iterated greedy algorithm for the permutation flowshop scheduling problem. European Journal of Operational Research, (177), 2033-2049. https://doi.org/10.1016/j.ejor.2005.12.009

\section{Copyrights}

Copyright for this article is retained by the author(s), with first publication rights granted to the journal.

This is an open-access article distributed under the terms and conditions of the Creative Commons Attribution license (http://creativecommons.org/licenses/by/4.0/). 